\title{
El estrés académico durante la emergencia sanitaria y sus afectaciones en las funciones básicas cognitivas: memoria, comprensión lenguaje, pensamiento
}

\section{Academic stress during the health emergency and its effects on basic cognitive functions: memory, language comprehension, thinking}

Luis René Indacochea Mendoza. ${ }^{1}$, Mónica Narciza López Pazmiño. ${ }^{2}$, Mariana Elizabeth Toasa Galarza. ${ }^{3} \&$ Katherine Estefanía Díaz Núñez. ${ }^{4}$

\begin{abstract}
.
Academic stress is a systemic, adaptive and psychological process, it is present in the impotence and uncertainty generated by different factors, increased burden of commitments, crowding of work, insufficient integration into the work environment, among other aspects. Aim. Analyze academic stress during the health emergency and its effects on basic cognitive functions: memory, language comprehension, thinking in adolescents who are in the sixth and seventh semesters of the Psychopedagogy Career of the Technical University of Ambato. Methodology. Cross-sectional mode, through the description, the frequency and distribution of the research proposed in a certain demographic group is evaluated, the field, descriptive and correlational modality was

1 Universidad Técnica de Ambato. Facultad de Ciencias Humanas y de la Educación. Carrera Psicopedagogía, Ambato, Ecuador, email luisrindacocheam@uta.edu.ec. https://orcid.org/0000-00020215-5263

${ }^{2}$ Universidad Tecnológica Indoamérica, Facultad de Ciencias Humanas y Desarrollo Social. Carrera Educación Básica. Ambato, Ecuador, email monilopez.2012@hotmail.es. https://orcid.org/0000-00027537-6630

3 Estudio Jurídico Legis Abogados. Ambato. Ecuador, email legis.abogados@hotmail.com. https://orcid.org/0000-0002-6447-3662

4 Universidad Católica del Ecuador Sede Ambato, Facultad de Psicología, Ambato, Ecuador, email kathye1996@gmail.com. https://orcid.org/0000-0002-0215-5263
\end{abstract}


applied, the documentary bibliographic information was included that allowed the establishment of a scientific base From the inquiry, the information gathering technique was the SISCO Academic Stress Inventory proposed by (Barraza, 2015) with a Cronbach Alpha of 0.90, a reliability that can be valued as very good; according to (Luján \& Cardona, 2015), a test that favored the knowledge of the level of stress presented by the students, together with a questionnaire, in the population we worked with a sample of 105 subjects object of analysis. Results. $30.48 \%$ present high stress, $40 \%$ have homework overload, $41.9 \%$ do not do work, $37.1 \%$ are restless in class, $37.14 \%$ do not attend teacher presentations, $40 \%$ are not responsible, $33.3 \%$ do not understand the topics, $28.6 \%$ do not participate in the classroom. Conclusion. Assessments, poor understanding of the topics addressed in content development, master classes, traditional pedagogy, make it difficult to strengthen attention, concentration, reasoning, the acquisition of abilities, skills and abilities, limiting performance and performance School.

Keywords: academic stress, health emergency, cognitive functions, performance, traditional pedagogy.

\section{Resumen.}

El estrés académico es un proceso sistémico, adaptativo y psicológico, está presente en la impotencia e incertidumbre generado por diferentes factores, aumento de la carga de compromisos, aglomeración de labores, insuficiente integración al ambiente de trabajo, entre otros aspectos. Objetivo. Analizar el estrés académico durante la emergencia sanitaria y sus afectaciones en las funciones básicas cognitivas: memoria, comprensión lenguaje, pensamiento en los adolescentes que cursan el sexto y séptimo semestre de la Carrera de Psicopedagogía de la Universidad Técnica de Ambato. Metodología. Modo transversal, a través de la descripción se evalúa la frecuencia y la distribución de la investigación planteada en un determinado grupo demográfico, se aplicó la modalidad de campo, descriptiva y correlacional, se incluyó la información bibliográfica documental que admitió el establecimiento de una base científica de la indagación, la técnica de recolección de información fue el Inventario de Estrés Académico SISCO propuesta por (Barraza, 2015) con un Alpha de Cronbach de 0.90, una confiabilidad que puede ser valorado muy bueno; según (Luján \& Cardona, 2015) test que favoreció en el conocimiento del nivel de estrés que presentaron los estudiantes, junto con un cuestionario, en la población se trabajó con una muestra de 105 sujetos objeto de análisis. Resultados. Presenta estrés alto (30,48\%), tiene sobrecarga de tareas (40\%), no realiza trabajos $(41,9 \%)$, es inquieto en la clase $(37,1 \%)$, no atiende a exposiciones de los docentes $(37,14 \%)$, no es responsable (40\%), no entiende las temáticas $(33,3 \%)$, no participa en el aula (28,6\%). Conclusión. Las evaluaciones, el escaso entendimiento de los temas abordados en el desarrollo de contenidos, las clases magistrales, la pedagogía tradicional, dificulta el fortalecimiento de la atención, concentración, el razonamiento, la adquisición de habilidades, destrezas y capacidades, limitando el rendimiento y desempeño escolar. 
Palabras claves: estrés académico, emergencia sanitaria, funciones cognitivas, desempeño, pedagogía tradicional.

\section{Introducción.}

La emergencia sanitaria denominada Covid 19, ha generado un retroceso en el ámbito, social, educativo, sobretodo ha impactado en el estado psicológico de los estudiantes, en especial en el sector universitario y diversos sectores vulnerables que han sido contagiados llegando en algunos casos a la muerte. Lamentablemente en el ámbito educativo fue complicado el aunar fuerzas para combatir y enfrentar la crisis, factor que impidió tomar las medidas apropiadas frente a situaciones de estrés; en busca de la vacuna se establecieron protocolos médicos, sin embargo el recorte de presupuestos destinados para reforzar la salud fue un desafío que enfrentaron los hospitales públicos y el sistema formativo.

El estrés a nivel mundial es un tema de preocupación que atrae la atención de diversos científicos quienes toman como objetivo el analizar la conducta humana, por esta razón en el análisis establecido por el (Bedoya \& Vasquez, 2019) afirma que afecta a la salud física y mental, al aumentar el riesgo de enfermedades (cáncer, herpes, gripe); además incrementa el peligro de contraer el coronavirus, y los trastornos digestivos. Para (Bucaram, García, Linzán, \& Reyes, 2019) son alarmantes las consecuencias que surgen del estrés en el área educativa, al acrecentar los problemas académicos, familiares o complicaciones en vida cotidiana.

Desde una revisión teórica sobre el estrés (Naranjo, 2009) menciona la dificultad en la atención y concentración y la insuficiente retención memorística (no sigue patrones lógicos); con respecto al ambiente educativo afecta a los adolescentes en el rendimiento académico, en las relaciones interpersonales, incrementa la violencia física y psicológica. Como expone (Jiménez, 2017) aumentan los miedos asociados, el fracaso y el incumplimiento de expectativas de padres de familia y docentes, evidenciándose síntomas de desmotivación y ansiedad, que afectan en el área emocional, cognitivo, conductual y fisiológico en el estudiantado (Pérez, Echauri, \& Salcedo, 2002), es decir influye de manera negativa en la eficiencia del aprendizaje.

En referencia al estrés y el aprendizaje en estudiantes universitarios (Duarte \& Fernández, 2015) afirman que el $88,1 \%$ presenta estrés frente a las diferentes tareas y actividades académicas, evidenciado en la exagerada preocupación o nerviosidad en escenarios educativos, donde exterioriza problemas de concentración, somnolencia (necesidad de dormir) y desinterés en el cumplimiento de trabajos; el estudio presentado tiene como finalidad analizar el estrés académico durante la emergencia sanitaria y sus afectaciones en las funciones básicas cognitivas: memoria, comprensión lenguaje, pensamiento; así, contribuye en la labor pedagógica en el proceso de formación. 


\section{El estrés tipología, y factores estresantes}

El estrés ilustra tiene su origen por el siglo XIV, observado en expresiones: adversidad, contrariedad, tensión, desasosiego, impaciencia, incertidumbre, ansiedad, malestar, angustia; Lazarus \& Folkman, (1986) afirma, en el siglo XVIII se reconoce en el área de la física como una alteración que repercute de forma negativa en las funciones cognitivas básicas; el autor indicado expone postulaciones teóricas mencionados por Pavlov (representante de las teorías de condicionamiento clásico), al establecer una relación entre un estímulo estresor y la respuesta fisiológica. Por lo tanto, el estrés es el resultado de las tensiones que presenta el individuo desde la infancia, incluye inconvenientes con los padres, descanso insuficiente, conflictos personales. Según Barreno, (2019) describe situaciones que generan conductas, pensamientos y reacciones a nivel físico y psicológico. Para Gonzales, (2016) es un conjunto de sensaciones desagradables producidas frente a contextos adversos.

Según manifiesta Jeréz \& Oyarzo (2015) el estrés es el conjunto de procesos y respuestas neuroendocrinas, inmunológicas, emocionales y conductuales que son percibidas por los sujetos como amenaza o peligro que afectan la integridad biológica o psicológica. Desde otra perspectiva Berrío \& Mazo, (2011) en el estrés figuran el nerviosismo, la tensión, el cansancio, el agobio, la inquietud y otras sensaciones similares, aspectos que incrementan la presión escolar. Por tanto, el estrés afecta en el área académica y emocional al generar una constante ansiedad dificultades de una concentración irritabilidad desmotivación aislamientos.

En su tipología el estrés incluye el eustrés que es positivo, a corto plazo, el sujeto efectúa actividades físicas, demuestra entusiasmo y creatividad, tiene capacidad de enfrentar situaciones complejas que aparecen durante el transcurso de su vida (Peinado, 2018). Distrés, es negativo, inicia en los reajustes o alteraciones en la rutina, aumenta el disgusto, la contradicción, y exterioriza escasa habilidad de adaptación que genera desequilibrio, de variaciones psicosomáticas, se evidencia en el malestar físico y emocional en el individuo (Briones, 2019). Por tal razón es trascendental la priorización en la planificación de contenidos, la utilización de diferentes técnicas de estudio, la alimentación saludable, la práctica del deporte, la relajación, el mantener el buen humor, la calma y tranquilidad.

En las fases del estrés Naranjo (2009) hace referencia a la reacción de alarma, donde la estructura corporal reconoce y se prepara para la acción mediante el enfrentamiento, en esta visión las glándulas endocrinas liberan hormonas que agrandan los latidos del corazón y el ritmo respiratorio aumentando la traspiración, evidenciando la dilatación de las pupilas, lo que provoca la escasa concentración Santamaría (2015). En la fase de resistencia el organismo repara las afectaciones generadas por la reacción de alarma, su energía es limitada para la adaptación, siendo significativo conservar la hiperactividad. 
En cuanto al agotamiento el cuerpo del sujeto exterioriza desmotivación e insuficiente disposición para el manejo adecuado de una situación.

\section{El estrés en el área académica y formativo, desde una visión pedagógica y psicológica}

Tomando como referencia las fases del estrés Acosta (2016) menciona, es trascendental fortalecer las capacidades para la detección de cambios estresantes que interfieren en el equilibrio emocional, aspecto que involucra el cerebro, (la razón, la mente, el intelecto); los nervios (estado emocional, freno inhibitorio), el corazón (flujo sanguíneo), las hormonas, el órgano digestivo y función muscular. Según Román \& Hernández (2011) en este ámbito argumentan, en el plano psicopedagógico es un proceso de cambio en los componentes de enseñanza y aprendizaje, que mediante mecanismos adaptativos exigen mejorar las experiencias en la formación académica; por tanto es una activación fisiológica, cognitiva y conductual ante estímulos y sucesos escolares. Desde el punto de vista del autor, el estado mental conlleva al cansancio, afectando en las habilidades de pensamiento, razonamiento y reflexión en el momento de consolidar los saberes.

Los estresores académicos son estímulos que fuerzan y sobrecargan al estudiante en el cumplimiento de una actividad en el ámbito educativo. Rodríguez (2014) expone los siguientes: sobrecarga de tareas (escritos, informes, exposiciones, demostraciones o recapitulaciones), insuficiente tiempo para efectuar los trabajos, evaluaciones permanentes, variedad de materias que estudia, aspectos que generan barreras que limitan la concentración y atención para las clases Caldera \& Pulido (2020); además los insuficientes conocimientos de los maestros en el manejo de metodologías, estrategias didácticas, intervenciones en público, el clima inadecuado para el aprendizaje, las competencias en grupo, el exceso de compromiso y responsabilidad, son conflictos deterioran las capacidades cognitivas.

Para Feldman, Goncalves, Chacón, Zaragoza, \& Bagés (2008) los escolares que enfrentan desafíos y retos requieren la aplicación de materiales físicos y psicológicos que disminuyan el agotamiento. Arribas (2011) menciona, entre las causas del estrés académico: la exagerada designación de tareas escolares y extraescolares, el cumplimiento con las evaluaciones, la sobrecarga de intervenciones y participaciones durante las clases orales, el escaso tiempo para efectuar tareas y entregar los trabajos, dificultades en la comprensión y entendimiento de temas o aspectos que indica el profesor durante la jornada educativa. Es decir los estudiantes universitarios al presentar síntomas como dolores de cabeza, dificultades en la digestión, sudoración, desconcentración, ansiedad y absentismo en las clases están padeciendo estrés académico.

Montes, (2012) incluye que la apreciación del educando al docente, motiva o desmotiva en el aprendizaje, así incluye una educación rutinaria donde el proceso académico es tradicional y memorístico (afecta al desarrollo cognitivo) las tareas con un tiempo limitado para su realización repercute en la concentración; la metodología inadecuada, las insuficientes estrategias disminuyen el rendimiento escolar, conllevando a una 
enseñanza pasiva que aumenta la presión en los estudiantes, aspectos que generan barreras en la autodeterminación, la toma de decisiones la valoración de sus posibilidades para alcanzar las metas propuestas.

Para Rosales (2016) cuando el estudiante se encuentra estresado el organismo incrementa la producción de hormonas (cortisol y adrenalina) que repercuten en las funciones que cumple el corazón, el nerviosismo arterial, y la actividad física, conllevando a un déficit en la concentración, fatiga intelectual, indiferencia, desatención, debilidad, nerviosismo, angustia, irritación, desvelo, tensión muscular, dolor de cabeza, agotamiento crónico, acrecentamiento o pérdida de peso, convulsiones, tics nerviosos, incertidumbre, insuficiente confianza en sí mismo, olvidos y problemas de digestión (Alfonso, Calcines, Monteagudo, \& Nieves, 2015). Así ante una complicación para la Psicología como es el estrés, y especialmente en el contorno académico en la enseñanza media superior, el exceso de responsabilidad por cumplir las obligaciones escolares, ha incrementado el bajo rendimiento académico

\section{Importancia en el desarrollo de las funciones cognitivas básicas frente a la emergencia sanitaria en tiempos vulnerables.}

Las funciones cognitivas según Feuerstein (1980) son consideradas necesarias en el aprendizaje, a su vez facilitan la recuperación del conocimiento; su función radica en la capacidad de aprender, recordar información, constituir, proyectar y solucionar problemas; mediante la concentración, atención, entendimiento y empleo del lenguaje. Al ser parte del sistema nervioso beneficia en la adquisición de experiencias previas y en su adaptación a nuevas situaciones.

Desde esta perspectiva, cumplen un papel primordial en la cotidianidad del hombre, en este sentido sobresale la sensación producida por medio de los sentidos; la percepción permite acopiar, almacenar, recolectar e interpretar información del entorno mediante los registros sensoriales. Por lo expuesto Pazmiño, Núñez, López, \& Pazmiño(2019) afirma, la percepción es un proceso constructivo que antes de procesar la nueva información, construye un esquema informativo que admite contrastar el estímulo, aceptar o rechazar; la atención promueve la selección de información sensorial, dirige los procesos mentales; la memoria, admite la codificación, almacenamiento y recuperación de la información.

Continuando con las funciones cognitivas, la motivación refuerza el esfuerzo por alcanzar las metas con la finalidad de satisfacer alguna necesidad individual; la comprensión está determinada la motivación e interés. El lenguaje humano, se basa en la capacidad para comunicar mediante signos lingüísticos, gestos y señas; la orientación consolida la conciencia en relación con el tiempo, el espacio y la persona. Funciones ejecutivas

Por otro lado Lesak, (1982) afirma. Las funciones ejecutivas son destrezas vinculadas con la conducta compleja, responde a situaciones que incluyen la determinación de objetivos, 
planeación de actividades, ejecución de acciones propositivas que conllevan al cumplimiento de los propósito, de esta forma aporta en el desempeño eficiente y productivo. En cuanto al pensamiento (Mejía \& Escobar, 2012) mencionan que transforma la información en la memoria al establecer conexiones entre ideas o representaciones. Por lo expuesto, Valdizán (2008) las funciones cognitivas básicas incluyen la percepción, el procesamiento, la organización, interpretación y almacenamiento de información.

\section{Metodologia.}

En el estudio, el trabajo es de corte transversal, (mediante la observación analiza datos de variables recopiladas en un periodo especifico de tiempo sobre una población, muestra o subconjunto predefinido) (Sampieri, 2003) el enfoque es mixto o cuanti-cualitativo, responde a la problemática, además mediante la cuantificación de resultados se efectúa el análisis descriptivo causa - efecto. La modalidad bibliográfica-documental tuvo el propósito de detectar, ampliar y profundizar diferentes enfoques, teorías conceptos o definiciones, que sustentaron el aspecto teórico. La indagación de campo promovió la recolección de datos e información de forma directa, al emplearse herramientas virtuales (Google Forms) que facilitaron el trabajo de campo

En cuanto al nivel o tipo de investigación es descriptiva, según (Sampieri, 2003) el trabajo se efectúa sobre realidades de hechos, aspecto que beneficia en la medición para comparar las causas y los efectos del estudio. Con respecto a la investigación correlacional (Sampieri, 2003) manifiesta que asocia variables mediante un patrón predecible para un grupo o población, busca conocer la relación o grado de asociación; en el estudio indaga el nivel de relación entre el estrés académico durante la emergencia sanitaria y sus afectaciones en las funciones básicas cognitivas: memoria, comprensión lenguaje, pensamiento.

\section{Participantes}

En cuanto a la población, se conformó por los adolescentes de 6to., y 7mo. Semestre, que cursan la carrera de Psicopedagogía en la Universidad Técnica de Ambato.

\section{Técnicas e instrumentos}

En los instrumentos se emplea el inventario SISCO del Estrés Académico aplicado por (Barraza, 2007), con el objetivo: reconocer las características del estrés que suele acompañar a durante los estudios académicos en la emergencia sanitaria; reporta una confiabilidad en alfa de Cronbach de 0.87, contiene treinta y uno ítems (un ítem, filtro en términos dicotómicos (si-no); un ítem; el nivel de intensidad del estrés académico es establecido en la escala Likert; ocho ítems equiparan los estresores académicos, quince se enfocan en los síntomas al estresor; seis ítems referentes a las estrategias de 
afrontamiento. Se aplica la escala Likert, con 5 categorías, cada una con su respectivo valor: nunca (1), rara vez (2), algunas veces (3), casi siempre (4), y siempre (5), (Ortega, 2018)

Tabla 1. Inventario SISCO del estrés académico

N.

ítems

\section{Estímulos Estresores}

1 Siente preocupación y nerviosismo

2 La competencia con los compañeros del grupo

3 Sobrecarga de tareas y trabajos escolares

4 La personalidad y el carácter del profesor

5 Las evaluaciones de los profesores (exámenes, ensayos, trabajos de investigación, etc.)

6 El tipo de trabajo que te piden los profesores (consulta de temas, fichas de trabajo, ensayos, mapas conceptuales, etc.)

$7 \quad$ No entender los temas que se abordan en la clase

8 Participación en clase (responder a preguntas, exposiciones, etc.)

9 Tiempo limitado para hacer el trabajo

\begin{tabular}{|c|c|}
\hline \multicolumn{2}{|r|}{ Reacciones Físicas } \\
\hline 10 & Trastornos en el sueño (insomnio o pesadillas) \\
\hline 11 & Fatiga crónica (cansancio permanente) \\
\hline 12 & Dolores de cabeza o migrañas \\
\hline 13 & Problemas de digestión, dolor abdominal o diarrea \\
\hline 14 & Rascarse, morderse las uñas, frotarse, etc. \\
\hline 15 & Somnolencia o mayor necesidad de dormir \\
\hline \multicolumn{2}{|r|}{ Reacciones Psicológicas } \\
\hline 16 & Inquietud (incapacidad de relajarse y estar tranquilo) \\
\hline 17 & Sentimientos de depresión y tristeza (decaído) \\
\hline 18 & Ansiedad, angustia o desesperación. \\
\hline 19 & Problemas de concentración \\
\hline 20 & Sentimiento de agresividad o aumento de irritabilidad \\
\hline 21 & Reacciones comportamentales \\
\hline 22 & Conflictos o tendencia a polemizar o discutir \\
\hline 23 & Aislamiento de los demás \\
\hline 24 & Desgano para realizar las labores escolares \\
\hline 25 & Aumento o reducción del consumo de alimentos \\
\hline \multicolumn{2}{|r|}{ Enfrentamiento } \\
\hline 26 & Habilidad asertiva (defender las preferencias ideas o sentimientos sin dañar a otros) \\
\hline 27 & Elaboración de un plan y ejecución de sus tareas \\
\hline 28 & Elogios a sí mismo \\
\hline 29 & La religiosidad (oraciones o asistencia a misa) \\
\hline 30 & Búsqueda de información sobre la situación \\
\hline 31 & Ventilación y confidencias (verbalización de las circunstancias ) \\
\hline
\end{tabular}

Fuente: (Ortega, 2018)

Compilado por: investigadores 
El coeficiente Alfa de Cronbach del instrumento asume un valor de 0,996 que se encuentra en el rango de lo aceptable y se considera como una confiabilidad estadísticamente significativa (Chapi, 2019)

Tabla 2. Estadísticos de fiabilidad. Inventario SISCO del estrés académico

\section{Estadísticos de fiabilidad}

Alfa de Cronbach N de elementos

,996

Fuente: estadísticos de fiabilidad

Elaborado por: investigadores

Se emplea un cuestionario semi-estructurado que beneficia en la adquisición de información relevante concerniente a las funciones cognitivas básicas, según Ruiz (1995), para elaborar el instrumento, se toma se considera las dimensiones (aprendizaje, sensación, percepción, atención, memoria), indicadores e ítems básicos; en este estudio se considera el estrés académico junto con las funciones cognitivas, consta de 13 ítems, comprende las alternativas: siempre (4), a veces (3), rara vez (2), nunca (1).

Tabla 3. Funciones Cognitivas Básicas

\begin{tabular}{|c|c|}
\hline N. & Ítems \\
\hline & Aprendizaje \\
\hline 1 & ¿Cuándo usted se siente cansado logra tener un aprendizaje significativo? \\
\hline 2 & ¿Cree usted que la metodología docente interfiere en el proceso de aprendizaje? \\
\hline \multirow[t]{2}{*}{3} & ¿Cree usted que la educación rutinaria genera un tipo de desajuste en su vida estudiantil? \\
\hline & Sensación \\
\hline 4 & $\begin{array}{l}\text { ¿Logra captar con precisión técnicas de aprendizaje representadas en imágenes como mapas } \\
\text { mentales? }\end{array}$ \\
\hline \multirow[t]{2}{*}{5} & ¿Aprende de manera rápida y significativa cuando manipula objetos al momento de estudiar? \\
\hline & Percepción \\
\hline 6 & $\begin{array}{l}\text { ¿Alguna vez ha discutido con un compañero o docente por algún trabajo que no entendió cómo } \\
\text { hacer? }\end{array}$ \\
\hline 7 & ¿Es capaz de crear conceptos relacionados a un tema que ha leídorecientemente? \\
\hline \multirow[t]{2}{*}{8} & $\begin{array}{l}\text { ¿Cuándo está usted con desgano de hacer alguna actividad tiende a leer en voz alta para entender } \\
\text { lo que debe hacer? }\end{array}$ \\
\hline & Atención \\
\hline 9 & ¿Los problemas familiares o emocionales afectan tu atención en clases? \\
\hline \multirow[t]{2}{*}{10} & ¿Se distrae con facilidad frente a estímulos visuales? \\
\hline & Memoria \\
\hline 11 & ¿Me cuesta recordar información que aprendí en la clase anterior? \\
\hline 12 & ¿Logro realizar una evaluación exitosamente después de culminar laclase? \\
\hline 13 & ¿Cuándo me piden alguna información logro recordar donde seencuentra? \\
\hline
\end{tabular}

Fuente: (Ortega, 2018)

Elaborado por: Investigadores 
La validación del instrumento se efectuó mediante el juicio valorativo de expertos ( $\mathrm{Mg}$. Carina Ayala, Psicóloga Clínica). Mientras la aprobación se efectuó mediante el Coeficiente Alfa de Cronbach, equivalente al 0,98 información fiable obtenida a través de mediciones sólidas y consistentes, Villanueva,(2016) menciona que el valor de fiabilidad para la investigación básica entre 0.7 y 0.8 ; en investigación aplicada sobre 0.95 , es excelente, mientras el coeficiente 0,8 es bueno.

Tabla 4. Estadísticos de fiabilidad. Funciones Cognitivas Básicas

\begin{tabular}{c} 
Estadísticos de fiabilidad \\
\hline Alfa de Cronbach
\end{tabular}

Fuente: estadísticos de fiabilidad

Elaborado por: investigadores

\section{Resultados.}

Se realizó el análisis e interpretación de datos estadísticos descriptivos con enfoque crítico y argumentativo, mediante cuatro ítems que facilitaron la asociación entre las variables, obteniendo los siguientes resultados: el 55,2\% siente nerviosismo y se distrae con provocaciones visuales, mientras el $21,9 \%$ casi no presenta expresiones de alteración; por otro lado, el $20 \%$ se distrae con facilidad ante estos tentaciones visuales, y finalmente el 2,9\% no se distrae; en referencia a la fatiga crónica, el 38,1\% varias veces siente esta reacción física, el 23,8\% alguna vez ha exteriorizado la sintomatología, mientras el $21 \%$ rara vez expresa el malestar, y el 9,5 nunca y el 7,6\% siempre demuestra un cansancio permanente.

En referencia a la fatiga crónica que afecta en el aprendizaje significativo un 39,8\% demuestra cansancio, el 31,1\% a veces integran la teoría y la práctica, el 25,2\% nunca alcanza el aprendizaje significativo, finalmente un 3,9\% siempre adquiere experiencias significativas aun cuando se siente cansado; el 20\% siempre se distrae con facilidad. El 92,92\% demuestra preocupación y nerviosismo en el ámbito académico, generado por entrega de tareas, exposiciones, o trabajos. El 39,05\% exterioriza un nivel de estrés medio, mientras el 30,48\% medianamente alto, un 20,95\% un nivel alto, el 7,62 \% medianamente bajo y finalmente un $1,90 \%$ bajo. En referencia a la competencia con los compañeros del grupo un $28,6 \%$ a veces, el 18,1\% nunca, el 12,4\% casi siempre; en la sobrecarga de tareas y trabajos escolares: el 41,9\% responden que casi siempre; en la personalidad y el carácter del docente el $37,1 \%$ afirman que a veces influye en el proceso de enseñanza.

En referencia a las evaluaciones mediante exámenes, ensayos, trabajos de investigación el $37,14 \%$ casi siempre se preocupa debido a su complejidad; en cuanto a las consultas 
de temas, fichas de trabajo, ensayos, mapas conceptuales, el 31,4\% casi siempre se obsesiona, mientras el 33,3\% no entiende los diferentes temas expuestos en el aula debido al desinterés, insuficiente motivación, deficiente utilización de metodología apropiada; por otra parte, el 33,3\% algunas veces participan en clases a preguntas, exposiciones, el $34,3 \%$ algunas veces presentan estrés debido al tiempo restringido para efectuar las tareas de aula, actividades o pruebas.

Entre otros resultados se obtiene que el 33,3\% padece trastornos en el sueño; por otra parte, el 32,4\% rara vez siente sueño; el 29,5\% exterioriza cierto tipo de reacción física como dolores de cabeza o migrañas; en el 30,5\% se denota un decaimiento y sentimientos de tristeza; el 37,1\% exterioriza rasgos de ansiedad, como desesperación y angustia; el $39 \%$ exterioriza problemas de concentración, el 28,6\% muestran emociones agresivas o irritabilidad en el contexto académico.

Por otro lado el $40 \%$ tiene una tendencia a tendencia a discutir o generar conflictos, sobre todo por la inadecuada organización en grupos de trabajo, el 30,5\% prefieren aislarse de los demás; un 34,3\% exterioriza un inapetencia al cumplir tareas académicas por la falta de interés hacia la asignatura, por la apatía de plasmar algún trabajo o una desmotivación por relaciones en el área social o familiar; el 37,1\% acrecienta o disminuye el consumo alimenticio, debido al corto para comer o por el escaso apetito debido a sus preocupaciones.

En el análisis estadístico se aplicó el Chi cuadrado, combinando las frecuencias mediante la selección de 4 interrogantes partiendo del inventario y el cuestionario, 2 para cada variable, por lo expuesto en el planteamiento de la hipótesis se empleó el Modelo lógico con probabilidad de aceptar o rechazar la Hipótesis nula $\mathrm{H}_{0}$ : El estrés académico no incide en las funciones cognitivas básicas, junto con la Hipótesis alterna $\mathrm{H}_{1}$ : El estrés académico si incide en las funciones cognitivas básicas en los educandos de 6to., y 7mo., semestre que cursan la Carrera de Psicopedagogía en la Universidad Técnica de Ambato.

Para la verificación de la presente hipótesis se utiliza el estadígrafo de Chi Cuadrado, que admitió la obtención de datos numéricos y estadísticos con exactitud a través de la ficha de observación aplicada a los adolescentes.

Tabla 5. Resumen del procesamiento de los casos

\begin{tabular}{|c|c|c|c|c|c|c|}
\hline \multicolumn{7}{|c|}{ Resumen del procesamiento de los casos } \\
\hline & \multicolumn{6}{|c|}{ Casos } \\
\hline & \multicolumn{2}{|c|}{ Válidos } & \multicolumn{2}{|c|}{ Perdidos } & \multicolumn{2}{|c|}{ Total } \\
\hline & $\mathrm{N}$ & Porcentaje & $\mathrm{N}$ & Porcentaje & $\mathrm{N}$ & Porcentaje \\
\hline $\begin{array}{l}\text { Estrés Académico } \\
* \text { funciones } \\
\text { cognitivas }\end{array}$ & 105 & $100,0 \%$ & 0 & $0,0 \%$ & 105 & $100,0 \%$ \\
\hline
\end{tabular}

Fuente: procesamiento de los casos 
En la verificación de la hipótesis se manejó el modelo lógico, mientras en el modelo estadístico se aplicó el cálculo del chi cuadrado con un error del 5\%, un nivel de significación del 0,05, una confiabilidad del 95\%, obteniendo en el resumen de procesamiento 10 '5 casos válidos, equivalente al 100\%

Tabla 6. Pruebas de chi-cuadrado

\begin{tabular}{|c|c|c|c|}
\hline \multicolumn{4}{|c|}{ Pruebas de chi-cuadrado } \\
\hline \multirow[b]{2}{*}{ Chi-cuadrado de Pearson } & Valor & $\mathrm{gl}$ & $\begin{array}{l}\text { Sig. asintótica } \\
\text { (bilateral) }\end{array}$ \\
\hline & $2225,069^{\mathrm{a}}$ & 1353 &, 000 \\
\hline Razón de verosimilitudes & 580,877 & 1353 & 1,000 \\
\hline Asociación lineal por lineal & 98,942 & 1 &, 000 \\
\hline $\mathrm{N}$ de casos válidos & \multicolumn{2}{|l|}{105} & \\
\hline \multicolumn{4}{|c|}{$\begin{array}{l}\text { a. } 1428 \text { casillas }(100,0 \%) \text { tienen una frecuencia esperada inferior a } 5 \text {. La frecuencia mínima esperada } \\
\text { es }, 01 \text {. }\end{array}$} \\
\hline
\end{tabular}

Fuente: pruebas de chi-cuadrado

Tabla 7. Resumen de prueba de hipótesis

Resumen de prueba de hipótesis

\begin{tabular}{|c|c|c|c|}
\hline Hipótesis nula & Test & Sig. & Decisión \\
\hline $\begin{array}{l}1 \text { La mediana de las } \\
\text { diferencias entre } \\
\text { estrés académico y } \\
\text { funciones básicas } \\
\text { cognitivas es igual a } 0 .\end{array}$ & $\begin{array}{l}\text { Prueba de Wilcoxon de } \\
\text { los rangos con signo de } \\
\text { muestras relacionadas }\end{array}$ & 0,14 & $\begin{array}{l}\text { Rechazar la } \\
\text { Hipótesis nula }\end{array}$ \\
\hline
\end{tabular}

Se muestran las significancias asintóticas. El nivel de significancia es 0,5

Fuente: resumen de prueba de hipótesis

En las Pruebas de chi-cuadrado la Significación Asintótica (bilateral) junto con la asociación lineal por lineal tiene un equivalente, 000 es inferior a 0,05. Es decir, se excluye la hipótesis nula y se admite la hipótesis alternativa

La aplicación de la Prueba de Wilcoxon afirma el rechazo de la hipótesis nula, y la aceptación de la hipótesis alternativa Hipótesis alterna $\mathrm{H}_{1}$ : El estrés académico sí incide en las funciones cognitivas básicas en los educandos de 6to., y $7 \mathrm{mo}$. semestre de la Carrera de Psicopedagogía de la Universidad Técnica de Ambato.

\section{Discusión}

Berrío \& Mazo (2011) el estrés se considera como un problema epidemiológico de elevada importancia a nivel mundial, manifestándose en todos los medios y escenarios de la actividad humana. Bhat, Tripathi, \& Kumar (2010), en el caso particular del estrés académico, se puede diferenciar como aquel que se desarrolla y expresa en el ámbito educativo, identificándose estrechamente vinculado a la valoración que realizan los 
adolescentes de las diferentes demandas o estresores de su entorno, que desequilibran su medio interno, así como las estrategias de afrontamiento para restablecer la homeostasis.

Para López, García, \& Cardona, 2012) (2012) la personalidad del docente puede jugar un importante papel dentro del desarrollo emocional, la empatía y el rendimiento académico de los estudiantes, considerando que la actividad rectora del desarrollo psicológico al final de la adolescencia comienza a orientarse hacia el estudio y la selección de la profesión donde el mérito académico del docente juega el rol de un ideal a alcanzar por este segmento poblacional lo que puede también sustentar lo observado en este trabajo. Quito, Tamayo, Buñay, \& Neira, (2017) entre los factores estresores identificados con mayor frecuencia se encuentran en orden de relevancia la personalidad (identidad, particularidad, temperamento) o el carácter del maestro, la competencia con los compañeros del grupo, la participación en clases y las evaluaciones (Correa, 2020). En menor medida se encontraron la falta de tiempo y la sobrecarga de tareas (Dias \& Hussain, 2011). La competencia con los compañeros así como la participación en clases y las evaluaciones se relaciona mucho con la obtención de calificaciones satisfactorias así como la promoción de curso, lo que puede explicar su influencia sobre el estado de ansiedad de los jóvenes.

Puescas, Castro, Falloc, \& Díaz (2010) las reacciones físicas, psicológicas y comportamentales asociadas al estrés se presentaron en una magnitud significativa. La somnolencia es uno de los factores identificados en los síntomas físicos del estrés en los estudiantes consecuencia de los trastornos del sueño y de un descanso de poca calidad durante el tiempo de actividad (Gonzales, 2016). Los adolescentes necesitan dormir entre ocho a nueve horas, para mantenerse con energías y desempeñarse en las labores académicas, si no se cumple esta rutina se sentirá cansado, la capacidad de atención disminuirá, y por ende aparecerán niveles de estrés y ansiedad que le conducirán a bajar su rendimiento académico. (Berrío \& Mazo, 2011), las estrategias de afrontamiento al estrés constituyen un elemento muy importante en el retorno a la homeostasis del organismo ya que permite manejar las demandas estresantes dentro de la interacción individuo-ambiente, así como las emociones que genera

\section{Conclusiones.}

- Con las investigaciones realizadas se logró evidenciar que existe un alto porcentaje de jóvenes que muestran estrés académico que incide en las funciones cognitivas básicas como son memoria, meditación, inteligencia, meditación y sensación. La sobrecarga de trabajos, actividades y labores, la correspondencia con el profesorado, las evaluaciones, la insuficiente comprensión y escaso entendimiento, dificulta en la concentración.

- Se evidenció que la escasa contención y atención a influido en la realización y cumplimiento de tareas de forma correcta, factores que repercuten en la 
responsabilidad, el trabajo en el aula, la toma de decisiones apropiada y solución de problemas, aspectos relevantes que influyen de forma negativa en las funciones cognitivas básicas, en el rendimiento académico, el desempeño y la adquisición de experiencias y aprendizajes significativos.

- Se realizó la prueba del Chi cuadrado y la Prueba de Wilcoxon que permitieron evidenciar la existencia de una correlación entre el estrés académico y las funciones cognitivas básicas; de esta forma se identificó que los educandos presentan un nivel de estrés elevado que influye en las relaciones interpersonales, la formación de la personalidad, la atención, el lenguaje, el pensamiento creativo, argumentativo, el razonamiento lógico y la reflexión.

- En los factores enfocados en las funciones cognitivas básicas de los educandos es evidente que la inadecuada metodología aplicada en el proceso de aprendizaje de las diferentes asignaturas no se relaciona con los avances de la ciencia y la tecnología, componentes que al no ser reforzados continuaran afectando en el progreso de las competencias y potencialidades de los adolescentes; es decir se continuará practicando una pedagogía tradicional donde sobresalga las clases magistrales (transmisión de información), la recepción de conceptos y definiciones, y la memorización de contenidos, procesos y procedimientos.

\section{Referencias bibliográficas.}

Acosta, M. (2016). Estrés académico y su relación con la autoestima de los estudiates de la Universidad de Cañete. Obtenido de Repositorio UNE: http://repositorio.une.edu.pe/bitstream/handle/UNE/1999/TM\%20CEDu\%203501\%20M1\%20$\% 20$ Melchor\%20Acosta.pdf?sequence $=1 \&$ isAllowed $=y$

Aldana, S., Vereda, F., Hidalgo-Alvarez, R., \& de Vicente, J. (2016). Facile synthesis of magnetic agarose microfibers by directed selfassembly. Polymer, 93, 61-64.

Alfonso, B., Calcines, M., Monteagudo, R., \& Nieves, Z. (2015). Estrés académico. Edumecentro, 7(2), 163-178.

Arribas, J. (2011). Hacia un modelo causal de las dimensiones del estrés académico e estudiantes de enfermería. Revista Educación .

Barraza, A. (2007). Inventario SISCO del estrés académico.

Barraza, A. (2 de Febrero de 2015). El inventario SISCO del estrés académico. Universidad Pedagógica de Durango, 6.

Barreno, J. (2019). El estrés y su influencia en las funciones ejecutivas de los profesionales del Centro de Salud Cevallos TipoB. Obtenido de Repositorio UTI.

Bedoya, E., \& Vasquez, D. (2019). Estrés y funcionamiento cognitivo en estudiantes universitarios . Revista Chilena de Neuropsicología, 23-29. 
Berrío, N., \& Mazo, R. (2011). Estrés académico. Revista de Psicologia Universidad de Antioquía.

Berrío, N., \& Mazo, R. (2011). Estrés Académico. Revista de Psicología Universidad de Antioquia, 48-65.

Bhat, S., Tripathi, A., \& Kumar, A. (2010). Supermacroprous chitosan-agarose-gelatin cryogels. in vitro characterization and in vivo assesment for cartilage tissue engineering. Journal of the Royal Society Interface, 1-15.

Bossis, G., Marins, J., Kuzhir, P., Volkova, O., \& Zubarev, A. (2015). Functionalized microfibers for field-responsive materials and biological applications. Journal of Intelligent Material Systems and Structures, 1-9.

Briones, M. (2019). El estrés. Obtenido de http://repositorio.untumbes.edu.pe/bitstream/handle/UNITUMBES/1407/MIRIA M\%20LUCRECIA\%20BRIONES\%20VELASQUEZ.pdf?sequence=1

Bucaram, M., García, J., Linzán, M., \& Reyes, A. (2019). El estrés y su influencia en el proceso cognitivo de los estudiantes . Revista Atlante Cuadernos de Educación y Desarrollo.

Caldera, J., \& Pulido, B. (Diciembre de 2020). Niveles de estrés y rendimiento académico en estudiantes de la carrera de Psicología del Centro Universitario de Los Altos. Universidad de Guadalajara, 55, 105.

Chapi, S. (2019). El estrés académico de los estudiantes de primero a quinto semestre de la carrera de Educación Inicial de la Facultad de Ciencias Humanas y de la Educación de la Universidad. Quito, Ecuador: Universidad Central del Ecuador.

Correa, R. (8 de Abril de 2020). Propuestas de reactivación económica frente al Covid19 en el Ecuador. Universidad Católica de Loja, 12.

Cortés, J., Puig, J., Morales , J., \& Mendizábal, E. (2011). Hidrogeles nanoestructurados termosensibles sintetizados mediante polimerización en microemulsión inversa. Revista Mexicana de Ingeniería Química., 10(3), 513-520.

Dias, A., \& Hussain, A. (2011). A biotechnological perspective on the application of iron oxide magnetic colloids modified with polysaccharides. Biotechnology Advances , 29, 142-155.

Dias, A., Hussain, A., Marcos, A., \& Roque, A. (2011). A biotechnological perspective on the application of iron oxide magnetic colloids modified with polysaccharides. Biotechnology Advances 29, 29, 142-155.

Duarte, D., \& Fernández, E. (2015). Enfoques de aprendizaje en estudiantes universitarios y su relación con el rendimiento académico. Revista electrónica ecucare, 37-51.

Estrada Guerrero, R., Lemus Torres, D., Mendoza Anaya, D., \& Rodriguez Lugo, V. (2010). Hidrogeles poliméricos potencialmente aplicables en Agricultura. Revista Iberoamericana de Polímeros, 12(2), 76-87.

Feldman, L., Goncalves, L., Chacón, G., Zaragoza, J., \& Bagés, N. (2008). Relaciones entre estres acádemico, apoyo social, salud mental y rendimiento acádemico en estudiantes universitarios venezolanos. Universidad Psychology, 7(3), 739-751. Obtenido de https://core.ac.uk/download/pdf/26136697.pdf 
Feuerstein. (1980). Instrumental enrichment: an intervention program for cognitive modifiability. Baltimore.

García-Cerda, L., Rodríguez-Fernández, O., Betancourt-Galindo, R., Saldívar-Guerrero, R., \& Torres-Torres, M. (2003). Síntesis y propiedades de ferrofluidos de magnetita. Superficies y Vacío., 16(1), 28-31.

Gonzales, M. (2016). Manejo del estres. Innovación y cualificación .

Ilg, P. (2013). Stimuli-responsive hydrogels cross-linked by magnetic nanoparticles. Soft Matter, 9, 3465-3468.

Jaimes, R. (2008). Validación del inventario SISCO del estrés académico en adultos jóvenes de la Universidad Pontificia Bolivariana, Seccional Bucaramanga. Medellín, Colombia: Universidad Pontificia Bolivariana.

Jeréz, M., \& Oyarzo, C. (2015). Estrés academico en estudiantes del Departamento de Salud de la Universidad de Los Lagos Osorno. Revista Chilena de Neuropsiquiatría.

Jiménez, A. (2017). Efectos de las terapias ecuestres en personas con parálisis cerebral. Revista Española de Discapacidad. Universidad Politécnica de Madrid, 5(2), 171-184.

Obtenido

de https://www.cedd.net/redis/index.php/redis/article/view/279

Lazarus, R., \& Folkman, S. (1986). Estrés y procesos cognitivos. Barcelona.

Lesak, M. (1982). El problema de evaluar las funciones ejecutivas. Revista Internacional de Psicología, 14(1- 4$)$ Obtenido de https://doi.org/10.1080/00207598208247445

Lewitus, D., Branch, J., Smith, K., Callegari, G., Kohn, J., \& Neimark, A. (2011). Biohybrid carbon nanotube/agarose fibers for neural tissue engineering. Advanced Functional Materials, 21, 2624-2632.

Lin, Y.-S., Huang, K.-S., Yang, C.-H., Wang, C.-Y., Yang, Y.-S., Hsu, H.-C., . . T Tsai, C.-W. (2012). Microfluidic synthesis of microfibers for magnetic-responsive controlled drug release and cell culture. PLoS ONE, 7(3), 1-8.

López, R., García, L., \& Cardona, L. (2012). Relación entre el estilo de personalidad de los docentes de preescolar y la relación docente. Corporación Universitaria Lasallista, Caldas-Antoquia.

Luján, L., \& Cardona, C. (14 de Julio de 2015). Construcción y validación de escalas de medición en salud: revisión de propiedades psicométricas. Archivos de Medicina. iMedPub Journals, 11(31), 10.

Mejía, E., \& Escobar, H. (2012). Caracterización de procesos cognitivos de memoria,lenguaje y pensamiento, en estudiantes con bajo y alto rendimiento académico. Revista Diversitas - Perspectivas en Psicología, 123-138.

Montes, I. (2012). Investigación longitudinal de los hábitos de estudio en una cohorte de alumnos universitarios. Revista Lasallista de Investigación, 96-110.

Naranjo, M. (2009). Una revision teorica sobre el estrés y algunos aspectos relevantes de éste en el ambito educativo. Revista Educación, 171-190.

Ortega, C. (2018). Programa basado en técnicas lúdicas para disminuir la agresividad. Loja, Ecuador: Universidad Nacional de Loja. 
Pazmiño, J., Núñez, B., López, J., \& Pazmiño, M. (12 de Mayo de 2019). Inteligencia emocional: una competencia para fortalecer el desarrollo cognitivo, el pensamiento crítico, la toma de decisiones y el rendimiento académico. Ciencia Digital, $\quad 3(2), \quad 12 . \quad$ Obtenido de http://cienciadigital.org/revistacienciadigital2/index.php/CienciaDigital/article/vi ew/472

Peinado, A. (Febrero de 2018). El estrés academico en los estudiantes del ultimo año, estudio realizado con estudiantes del ultimo año del ciclo diversificado del Instituto Nacional de Educación Diversificada, San Pedro Sacatepéquez. Obtenido de Repositorio USAC: http://biblioteca.usac.edu.gt/tesis/29/29_0548.pdf

Pérez, J., Echauri, M., \& Salcedo, A. (2002). El estrés. Instituto de Salud Pública, 19. Obtenido de http://files.sld.cu/bmn/files/2014/07/estresfolleto.pdf

Puescas, P., Castro, B., Falloc, V., \& Díaz, C. (2010). Factores asociados al nivel de estrés previo un examen en estudiantes de Educación Secundaria en cuatro Instituciones Educativas. Revista Cuerpo Médico HNAAA, 88-93.

Quito, J., Tamayo, M., Buñay, D., \& Neira, O. (2017). Estrés académico en estudiantes de tercero de bachillerato de unidades educactivas particuales del Ecuador. Revista Electrónica de Psicologia Iztacala, 253-276.

Rodríguez, B. (2014). Estresores académicos percibidos por estudiantes pertenecientes a la Escuela de Enfermeria de Ávila Centro Adscrito a la Universidad de Salamanca. Revista Enfermería CyL, 6(2).

Román, C., \& Hernández, J. (2011). El estrés académico: Una revisión crítica del concepto desde las ciencias de la educación. Revista electrónica de Psicología Iztacala, 14(2).

Rosales, J. (2016). Estrés académico y hábitosde estudio en universitarios de la carrera de psicologia de un centro de formacion superior privada de Lima-Sur. Obtenido de

http://repositorio.autonoma.edu.pe/bitstream/AUTONOMA/124/1/ROSALES\% 20FERNANDEZ.pdf

Ruiz Estrada, G. (2004). Desarrollo de un Sistema de liberación de fármacos basado en nanopartículas magnéticas recubiertas con Polietilénglicol para el tratamiento de diferentes enfermedades. Madrid: Universidad Autónoma de Madrid. Departamento de Física Aplicada.

Ruiz, L. (1995). Competencia motriz : elementos para compender el aprendizaje motor en educación física escolar. Madrid: Gymnos.

Sampieri, R. H. (2003). Metodología de la investigación (Vol. VI). (M. I. Martínez, Ed.) México, México: Interamericana.

Sampieri, R. H. (2003). Metodología de la investigación (Vol. VI). (M. I. Martínez, Ed.) México, México: Interamericana.

Santamaría, S. (2015). Estudio comparativo de factores estresantes y el nivel de agotamiento emocional en los médicos que laboran en el Hospital Municipal de Ambato. Ambato, Tungurahua, Ecuador: Universidad Técnica de Ambato. 
Song, J., King, S., Yoon , S., Cho, D., \& Jeong, Y. (2014). Enhanced spinnability of narbon nanotube fibers by surfactant addition. Fiberes and Polymers, 15(4), 762766.

Tartaj, P., Morales, M., González-Carreño, T., Veintemillas-Verdaguer, S., \& Serna, C. (2005). Advances in magnetic nanoparticles for biotechnology applications. Journal of Magnetism and Magnetic Materials, 290, 28-34.

Valdizán, J. (2008). Funciones cognitivas y redes neuronales del cerebro social . Revista de neurologia , 65-68.

Villanueva, S. (24 de Octubre de 2016). Alfa Cronbach, Prueba de regresión lineal, Prueba T, Chi cuadrada \& Coeficiente de correlación de Pearson. Pedagogía, 12.

Wulff-Pérez , M., Martín-Rodriguez, A., Gálvez-Ruiz, M., \& de Vicente, J. ( 2013 ). The effect of polymer surfactant on the rheological properties of nanoemulsions. Colloid and Polymer Science, 291, 709-716.

Zamora Mora, V., Soares, P., Echeverria, C., Hernández , R., \& Mijangos, C. (2015). Composite chitosan/Agarose ferrogels for potential applications in magnetic hyperethermia. Gels., 1, 69-80. 
PARA CITAR EL ARTÍCULO INDEXADO.

Indacochea Mendoza, L. R., López Pazmiño, M. N., Toasa Galarza, M. E., \& Díaz Núñez, K. E. (2021). El estrés académico durante la emergencia sanitaria y sus afectaciones en las funciones básicas cognitivas: memoria, comprensión lenguaje, pensamiento. Explorador Digital, 5(2), 101-119. https://doi.org/10.33262/exploradordigital.v5i2.1664

\section{¿Ciencia}

El artículo que se publica es de exclusiva responsabilidad de los autores y no necesariamente reflejan el pensamiento de la Revista Explorador Digital.

El artículo queda en propiedad de la revista y, por tanto, su publicación parcial y/o total en otro medio tiene que ser autorizado por el director de la Revista Explorador Digital.
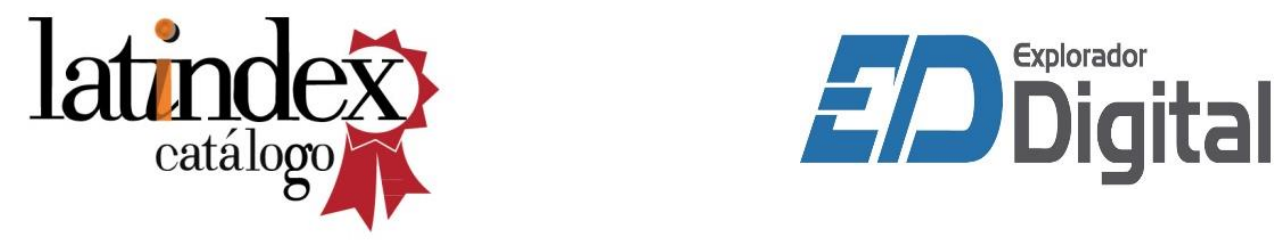